\title{
On the sum of the two largest Laplacian eigenvalues of unicyclic graphs
}

\author{
Yirong Zheng ${ }^{1,2^{*}}$, An Chang $^{2}$ and Jianxi Li2,3
}

\section{"Correspondence:}

yrzheng@xmut.edu.cn

${ }^{1}$ School of Applied Mathematics,

Xiamen University of Technology,

Xiamen, Fujian, P.R. China

${ }^{2}$ Center for Discrete Mathematics,

Fuzhou University, Fuzhou, Fujian, P.R. China

Full list of author information is available at the end of the article

\begin{abstract}
Let $G$ be a simple graph and $S_{2}(G)$ be the sum of the two largest Laplacian eigenvalues of $G$. Guan et al. (J. Inequal. Appl. 2014:242, 2014) determined the largest value for $S_{2}(T)$ among all trees of order $n$. They also conjectured that among all connected graphs of order $n$ with $m(n \leq m \leq 2 n-3)$ edges, $G_{m, n}$ is the unique graph which has maximal value of $S_{2}(G)$, where $G_{m, n}$ is a graph of order $n$ with $m$ edges which has $m-n+1$ triangles with a common edge and $2 n-m-3$ pendent edges incident with one end vertex of the common edge. In this paper, we confirm the conjecture with $m=n$.
\end{abstract}

MSC: $05 C 50 ; 15 A 48$

Keywords: Laplacian eigenvalue; sum of eigenvalues; largest eigenvalue; unicyclic graph

\section{Introduction}

Let $G=(V, E)$ be a simple connected graph with vertex set $V(G)$ and edge set $E(G)$. Its order is $|V(G)|$, denoted by $n(G)$ (or $n$ for short), and its size is $|E(G)|$, denoted by $m(G)$ (or $m$ for short). For a vertex $v \in V(G)$, let $N(v)$ be the set of all neighbors of $v$ in $G$ and let $d(v)=|N(v)|$ be the degree of $v$. Particularly, denote by $\Delta(G)$ the maximum degree of $G$. A pendent vertex is a vertex with degree one. The diameter of a connected graph $G$, denoted by $d(G)$, is the maximum distance among all pairs of vertices in G. Let $S_{n}$ and $P_{n}$ be the star and the path of order $n$, respectively. Let $S_{a, b}^{k}$ be the tree of order $n$ obtained from two stars $S_{a+1}, S_{b+1}$ by joining a path of length $k$ between their central vertices (see Figure 2). For all other notions and definitions, not given here, see, for example, [1] or [2] (for graph spectra).

Let $A(G)$ and $D(G)$ be the adjacency matrix and the diagonal matrix of vertex degrees of $G$, respectively. The matrix $L(G)=D(G)-A(G)$ is called Laplacian matrix of $G$. The Laplacian matrix is an important topic in the theory of graph spectra. We use the notation $I_{n}$ for the identity matrix of order $n$ and denote by $\phi(G, x)=\operatorname{det}\left(x I_{n}-L(G)\right)$ the Laplacian characteristic polynomial of $G$. It is well known that $L(G)$ is positive semidefinite symmetric and singular. Denote its eigenvalues by $\mu_{1}(G) \geq \mu_{2}(G) \geq \cdots \geq \mu_{n}(G)$ (or simply $\mu_{1} \geq \mu_{2} \geq \cdots \geq \mu_{n}$ sometimes for convenience) which are always enumerated in nonincreasing order and repeated according to their multiplicity. Note that each row sum of $L(G)$ is 0 and, therefore, $\mu_{n}(G)=0$. Fiedler [3] showed that the second smallest eigenvalue $\mu_{n-1}(G)$ of $L(G)$ is 0 if and only if $G$ is disconnected. Thus the second smallest eigenvalue

(c) 2015 Zheng et al. This article is distributed under the terms of the Creative Commons Attribution 4.0 International License (http://creativecommons.org/licenses/by/4.0/), which permits unrestricted use, distribution, and reproduction in any medium, provided you give appropriate credit to the original author(s) and the source, provide a link to the Creative Commons license, and indicate if changes were made. 
Figure $1 G_{n+1, n} . G_{m, n}$ is a graph of order $n$ with $m$ edges which has $m-n+1$ triangles with a common edge and $2 n-m-3$ pendent edges incident with one end vertex of the common edge.

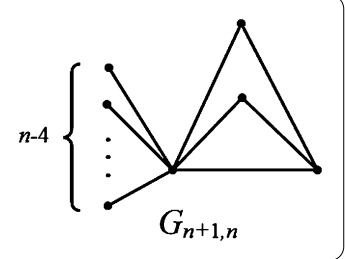

of $L(G)$ is popularly known as the algebraic connectivity of $G$. The largest eigenvalue $\mu_{1}(G)$ of $L(G)$ is usually called the Laplacian spectral radius of the graph $G$. Recently, some of the research has been focused on $\mu_{1}, \mu_{2}$ or $u_{n-1}$ (see [3-9]).

Let $S_{k}(G)=\sum_{i=1}^{i=k} \mu_{i}(G)$ be the sum of the $k$ largest Laplacian eigenvalues of $G$. Brouwer conjectured that $S_{k}(G) \leq m(G)+\left(\begin{array}{c}k+1 \\ 2\end{array}\right)$ for $k=1,2, \ldots, n$. The conjecture is still open, but some advances on the conjecture have been achieved (see [10-14]). Specially, for $k=2$, Haemers et al. [12] proved that $S_{2}(G) \leq m(G)+3$ for any graph $G$. When $G$ is a tree, Fritscher et al. [15] improved this bound by giving $S_{2}(T) \leq m(T)+3-\frac{2}{n(T)}$, which implies that Haemers' bound is always not attainable for trees. Therefore, it is interesting to determine which tree has maximal value of $S_{2}(T)$ among all trees of order $n$. Guan et al. [11] proved that $S_{2}(T) \leq S_{2}\left(T_{\left\lceil\frac{n-2}{2}\right\rceil,\left\lfloor\frac{n-2}{2}\right\rfloor}^{1}\right)$ for any tree of order $n \geq 4$, and the equality holds if and only if $T \cong T_{\left\lceil\frac{n-2}{2}\right\rceil,\left\lfloor\frac{n-2}{2}\right\rfloor}^{1}$. For any graph $G$ of order $n$ with $m$ edges, note that $\mu_{1}(G) \leq n(G)$. Then Haemers' bound is clearly not attainable when $2 n(G)<m(G)+3$. For $m(G)+3 \leq 2 n(G)$, Guan et al. [11] showed that $S_{2}\left(G_{m, n}\right)=m\left(G_{m, n}\right)+3$, where $G_{m, n}$ is a graph of order $n$ size $m$ which has $m-n+1$ triangles with a common edge and $2 n-m-3$ pendent edges incident with one end vertex of the common edge $\left(G_{n+1, n}\right.$ is illustrated in Figure 1). This indicates that Haemers' bound is always sharp for connected graphs $(m \leq 2 n-3)$ other than trees. The following conjecture on the uniqueness of the extremal graph is also presented in [11].

Conjecture 1.1 [11] Among all connected graphs of order $n$ with $m$ edges $(n \leq m \leq 2 n-3)$, $G_{m, n}$ is the unique graph with maximal value of $S_{2}(G)$, that is, $S_{2}\left(G_{m, n}\right)=m\left(G_{m, n}\right)+3$.

In this paper, we confirm Conjecture 1.1 with $m=n$.

\section{Preliminaries}

In this section, we present some lemmas which will be useful in the subsequent sections. For $\mu_{1}(G)$, the following results are well known.

Lemma 2.1 Let $G$ be a connected graph of order $n, d_{i}=d\left(v_{i}\right)$ and $m_{i}=\sum_{v_{j} \in N\left(v_{i}\right)} d_{j} / d_{i}$. Then

(1) [4] $\mu_{1}(G) \leq n(G)$ with equality if and only if the complement of $G$ is disconnected;

(2) $[8] \mu_{1}(G) \leq \max \left\{d_{i}+m_{i} \mid v_{i} \in V(G)\right\}$.

Lemma 2.2 [7] Let $G$ be a connected graph of order $n \geq 4$ with $m$ edges. Then

$$
\mu_{1}(G)<\max \left\{\Delta(G), m-\frac{n-1}{2}\right\}+2 .
$$

Lemma 2.3 [9] Let $T$ be a tree of order $n$ with $d(T) \geq 3$. Then $\mu_{1}(T)<n-0.5$.

Lemma 2.4 Let $T$ be a tree of order $n$ with $d(T) \geq 4$. Then $\mu_{1}(T)<n-1$. 
Proof For any tree $T$ of order $n$ with $d(T) \geq 4$, it follows that $n \geq 5$ and $\Delta(T) \leq n-3$. That is, $\Delta(T)+2 \leq n-1$ and $m-\frac{n-1}{2}+2=\frac{n-1}{2}+2 \leq n-1$. Then the result follows from Lemma 2.2.

The following theorem from matrix theory plays a key role in our proofs. We denote the eigenvalues of a symmetric matrix $M$ of order $n$ by $\lambda_{1}(M) \geq \lambda_{2}(M) \geq \cdots \geq \lambda_{n}(M)$.

Theorem 2.5 [16] Let $A$ and $B$ be two real symmetric matrices of size $n$. Then, for any $1 \leq k \leq n$,

$$
\sum_{i=1}^{k} \lambda_{i}(A+B) \leq \sum_{i=1}^{k} \lambda_{i}(A)+\sum_{i=1}^{k} \lambda_{i}(B) .
$$

From Theorem 2.5, the following lemma is immediate.

Lemma 2.6 Let $G_{1}, \ldots, G_{r}$ be some edge disjoint graphs. Then, for any $k$,

$$
S_{k}\left(G_{1} \cup \cdots \cup G_{r}\right) \leq \sum_{i=1}^{r} S_{k}\left(G_{i}\right) .
$$

The following lemma can be found in [2] and is well known as the interlacing theorem of Laplacian eigenvalues.

Lemma 2.7 [2] Let $G$ be a graph of order $n$ and let $G^{\prime}$ be the graph obtained from $G$ by inserting a new edge into $G$. Then the Laplacian eigenvalues of $G$ and $G^{\prime}$ interlace, that is,

$$
\mu_{1}\left(G^{\prime}\right) \geq \mu_{1}(G) \geq \cdots \mu_{n}\left(G^{\prime}\right) \geq \mu_{n}(G)=0 .
$$

Lemma 2.8 [2] Let $A$ be a symmetric matrix of order $n$ with eigenvalues $\lambda_{1} \geq \lambda_{2} \geq \cdots \geq \lambda_{n}$ and $B$ be a principal submatrix of $A$ of order $m$ with eigenvalues $\mu_{1} \geq \mu_{2} \geq \cdots \geq \mu_{m}$. Then the eigenvalues of $B$ interlace the eigenvalues of $A$, that is, $\lambda_{i} \geq \mu_{i} \geq \lambda_{n-m+i}$ for $i=1, \ldots, m$. Specially, for $v \in V(G)$, let $L_{v}(G)$ be the principal submatrix of $L(G)$ formed by deleting the row and column corresponding to vertex $v$, then the eigenvalues of $L_{v}(G)$ interlace the eigenvalues of $L(G)$.

Lemma 2.9 For any tree $T$ of order $n$, if there exists an edge $e \in E(T)$ such that $\min \left\{e\left(T_{1}\right), e\left(T_{2}\right)\right\} \geq 2$ and $\min \left\{d\left(T_{1}\right), d\left(T_{2}\right)\right\} \geq 3$ or $\max \left\{d\left(T_{1}\right), d\left(T_{2}\right)\right\} \geq 4$, then $S_{2}(T)<$ $n(T)+1$, where $T_{1}, T_{2}$ are the two components of $T-e$.

Proof Let $T-e=T_{1} \cup T_{2}$. By Lemma 2.6, it suffices to show that $S_{2}\left(T_{1} \cup T_{2}\right)<n(T)-1$. If $\mu_{1}(T)=\mu_{1}\left(T_{1}\right)$ and $\mu_{2}(T)=\mu_{2}\left(T_{1}\right)$ (or $\mu_{1}(T)=\mu_{1}\left(T_{2}\right)$ and $\mu_{2}(T)=\mu_{2}\left(T_{2}\right)$ ), then the result follows since $S_{2}\left(T_{1} \cup T_{2}\right)=S_{2}\left(T_{i}\right)<m\left(T_{i}\right)+3 \leq m(T)=n(T)-1$ for $i=1,2$. In what follows, we assume that $S_{2}\left(T_{1} \cup T_{2}\right)=\mu_{1}\left(T_{1}\right)+\mu_{1}\left(T_{2}\right)$. If $\min \left\{d\left(T_{1}\right), d\left(T_{2}\right)\right\} \geq 3$, then Lemma 2.3 implies that $S_{2}\left(T_{1} \cup T_{2}\right)<\left(n\left(T_{1}\right)-0.5\right)+\left(n\left(T_{2}\right)-0.5\right)=n(T)-1$. If $\max \left\{d\left(T_{1}\right), d\left(T_{2}\right)\right\} \geq 4$, say $d\left(T_{2}\right) \geq 4$, then Lemmas 2.1 and 2.4 imply that $S_{2}\left(T_{1} \cup T_{2}\right)<n\left(T_{1}\right)+\left(n\left(T_{2}\right)-1\right)=$ $n(T)-1$. This completes the proof.

Lemma 2.10 For any tree $T$ of order $n$ with $d(T)=5, S_{2}(T)<n(T)+1$. 

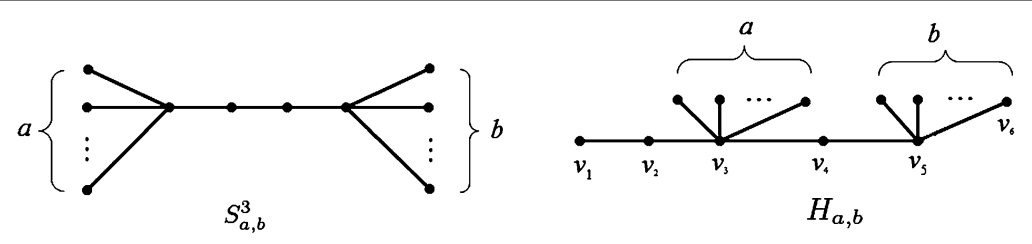

Figure 2 Two trees of order $n$.

Proof Without loss of generality, we assume that $v_{1} v_{2} v_{3} v_{4} v_{5} v_{6}$ is a path of length 5 in $T$. We now consider the following three cases.

Case $1 . \min \left\{d\left(v_{3}\right), d\left(v_{4}\right)\right\} \geq 3$.

Let $T_{1}, T_{2}$ be the two components of $T-v_{3} v_{4}$. Then the result follows from Lemma 2.9.

Case 2. $d\left(v_{3}\right)=d\left(v_{4}\right)=2$.

Note that $d(T)=5$. Then $T$ is isomorphic to $S_{a, b}^{3}$ (see Figure 2), where $a, b \geq 1$ and $a+$ $b+4=n$. By an elementary calculation, we have $\phi\left(S_{a, b}^{3}, \lambda\right)=\lambda(\lambda-1)^{n-5} f_{1}(\lambda)$, where $f_{1}(\lambda)=$ $\lambda^{4}-(n+3) \lambda^{3}+(5 n+a b-4) \lambda^{2}-(6 n+3 a b-10) \lambda+n$. Denoted by $\lambda_{1} \geq \lambda_{2} \geq \lambda_{3} \geq \lambda_{4}>0$ are the four roots of $f_{1}(\lambda)=0$. Then we have $\lambda_{1}+\lambda_{2}+\lambda_{3}+\lambda_{4}=n+3$ since $\sum_{i=1}^{i=n} \mu_{i}=2 m$. Note that $S_{a, b}^{3}$ contains $P_{6}$ as a subgraph. Then Lemma 2.7 implies that $\lambda_{3} \geq \mu_{3}\left(P_{6}\right)=2$. Thus, $S_{2}(T)=\lambda_{1}+\lambda_{2}=n+3-\left(\lambda_{3}+\lambda_{4}\right)<n+3-\lambda_{3}<n-1$, as desired.

Case 3. $d\left(v_{3}\right) \geq 3$ and $d\left(v_{4}\right)=2$ (or $d\left(v_{4}\right) \geq 3$ and $d\left(v_{3}\right)=2$ ).

Without loss of generality, we assume that $d\left(v_{3}\right) \geq 3$ and $d\left(v_{4}\right)=2$. If $d\left(v_{2}\right) \geq 3$, let $T-$ $v_{2} v_{3}=T_{1} \cup T_{2}$; if there is $P_{3}=u v v_{3}$ attached to $v_{3}$, let $T-v_{3} v_{4}=T_{1} \cup T_{2}$, where $u, v \neq$ $v_{i}(i=1,2, \ldots, 6)$. Then the result follows from Lemma 2.9. We now assume that $d\left(v_{2}\right)=$ $d\left(v_{4}\right)=2$ and all the neighbors of $v_{3}$ except for $v_{2}$ and $v_{4}$ are pendent vertices. That is, $T$ is isomorphic to $H_{a, b}$, where $H_{a, b}$ (see Figure 2) is the tree of order $n$ obtained from $v_{1} v_{2} v_{3} v_{4} v_{5} v_{6}$ by attaching $a$ and $b-1$ pendent vertices to $v_{3}$ and $v_{5}$, respectively, where $a, b \geq 1$ and $a+b+5=n$. Note that the matrix $1 \cdot I_{n}-L\left(H_{a, b}\right)$ has $a$ and $b$ different identical rows. Then the multiplicity of eigenvalue 1 is at least $n-7$. Let $\lambda_{1} \geq \lambda_{2} \geq \lambda_{3} \geq \lambda_{4} \geq \lambda_{5} \geq$ $\lambda_{6}>\lambda_{7}=0$ be the other seven eigenvalues. Then $\lambda_{1}+\lambda_{2}+\lambda_{3}+\lambda_{4}+\lambda_{5}+\lambda_{6}+\lambda_{7}=n+5$ since $\sum_{i=1}^{i=n} \mu_{i}=2 m$. For $a, b \geq 2, H_{a, b}$ contains $H_{2,2}$ as a subgraph. Then by Lemma 2.7 we have $\lambda_{3} \geq \mu_{3}\left(H_{2,2}\right)=2.44$ and $\lambda_{4} \geq \mu_{4}\left(H_{2,2}\right)=1.59$. Therefore, $S_{2}(T)=\lambda_{1}+\lambda_{2}<n+5-\left(\lambda_{3}+\right.$ $\left.\lambda_{4}\right)<n+1$, as required. If $a=1$, then by Lemmas 2.1 and 2.8 we have $\mu_{1}\left(H_{1, b}\right) \leq(n-5)+\frac{n-4}{n-5}$ and $\mu_{2}\left(H_{1, b}\right) \leq \mu_{1}\left(L_{v_{5}}\left(H_{1, b}\right)\right)=4.26$. That is, $S_{2}(T)=S_{2}\left(H_{1, b}\right)=\mu_{1}\left(H_{1, b}\right)+\mu_{2}\left(H_{1, b}\right)<n+1$, as required. Similarly, if $b=1$, then by Lemmas 2.1 and 2.8 we have $\mu_{1}\left(H_{a, 1}\right) \leq(n-4)+\frac{n-2}{n-4}$ and $\mu_{2}\left(H_{a, 1}\right) \leq \mu_{1}\left(L_{v_{3}}\left(H_{a, 1}\right)\right)=3.0$. It follows that $S_{2}(T)=S_{2}\left(H_{a, 1}\right)=\mu_{1}\left(H_{a, 1}\right)+\mu_{2}\left(H_{a, 1}\right)<n+1$, as required.

From the discussion above, the proof is completed.

Lemma 2.11 Let $T$ be a tree of order $n$ with $d(T) \geq 6$. Then $S_{2}(T)<n(T)+1$.

Proof We now consider the following two cases.

Case 1. $d(T) \geq 7$.

Let $v_{1} v_{2} v_{3} v_{4} v_{5} v_{6} v_{7} v_{8}$ be a path of length 7 in $T$ and $T-v_{4} v_{5}=T_{1} \cup T_{2}$. Then the result follows from Lemma 2.9.

Case 2. $d(T)=6$.

If $T=P_{7}$, then the result follows since $S_{2}\left(P_{7}\right)<8$. If $T \neq P_{7}$, let $v_{1} v_{2} v_{3} v_{4} v_{5} v_{6} v_{7}$ be a path of length 6 in $T$. If $d\left(v_{4}\right) \geq 3$, let $T-v_{3} v_{4}=T_{1} \cup T_{2}$; if $d\left(v_{3}\right) \geq 3$ (or $d\left(v_{5}\right) \geq 3$ ), let $T-v_{3} v_{4}=$ 
Figure 3 An example of firefly graph.

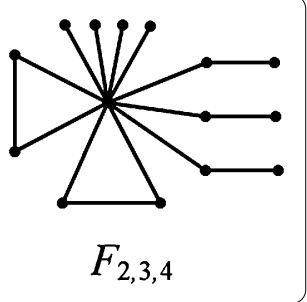

$T_{1} \cup T_{2}$ (or $T-v_{4} v_{5}=T_{1} \cup T_{2}$ ); if $d\left(v_{2}\right) \geq 3$ (or $d\left(v_{6}\right) \geq 3$ ), let $T-v_{2} v_{3}=T_{1} \cup T_{2}$ (or $\left.T-v_{5} v_{6}=T_{1} \cup T_{2}\right)$. In each of the above cases, by Lemma 2.9, we have $S_{2}(T)<n(T)+1$. This completes the proof.

A firefly graph $F_{s, t, n-2 s-2 t-1}(s \geq 0, t \geq 0$ and $n-2 s-2 t-1 \geq 0)$ is a graph with $n$ vertices that consists of $s$ triangles, $t$ pendent paths of length 2 and $n-2 s-2 t-1$ pendent edges, sharing a common vertex. An example of a firefly graph $F_{2,3,4}$ is illustrated in Figure 3 . Clearly $F_{0,0, n-1} \cong S_{n}$ and $F_{1,0, n-3} \cong G_{n, n}$.

Lemma 2.12 [6] The second largest Laplacian eigenvalue of $F_{1, t, n-2 t-3}$ satisfies $\mu_{2}\left(F_{1, t, n-2 t-3}\right)=3$.

Note that for $t \geq 1$, the complement of $F_{1, t, n-2 t-3}$ is connected. Hence Lemma 2.1 implies that $\mu_{1}\left(F_{1, t, n-2 t-3}\right)<n\left(F_{1, t, n-2 t-3}\right)$. This together with Lemma 2.12 implies the following lemma.

Lemma 2.13 For $t \geq 1, S_{2}\left(F_{1, t, n-2 t-3}\right)<n\left(F_{1, t, n-2 t-3}\right)+3$.

\section{Main result}

A unicyclic graph is a connected graph whose number of edges $m$ is equal to the number of vertices $n$. It is easy to see that each unicyclic graph can be obtained by attaching rooted trees to the vertices of a cycle $C_{k}$ for some $k$. Thus if $R_{1}, \ldots, R_{k}$ are $k$ rooted trees (of orders $n_{1}, \ldots, n_{k}$, say), then we adopt the notation $U\left(R_{1}, \ldots, R_{k}\right)$ to denote the unicyclic graph $G$ (of order $n=n_{1}+\cdots+n_{k}$ ) obtained by attaching the rooted tree $R_{i}$ to the vertex $v_{i}$ of a cycle $C_{k}=v_{1} v_{2} \cdots v_{k} v_{1}$ (i.e., by identifying the root of $R_{i}$ with the vertex $v_{i}$ for $i=1, \ldots, k$ ). Denote by $e\left(v_{i}\right)$ the maximum distance between $v_{i}$ and any vertex of $R_{i}$. In the special case when $R_{i}$ is a rooted star $K_{1, a_{i}}$ with the center of the star as its root (that is, $e\left(v_{i}\right)=1$ ), we will simplify the notation by replacing $R_{i}$ by the number $a_{i}$.

The following lemma is immediate from Lemmas 2.6, 2.10 and 2.11.

Lemma 3.1 For any unicyclic graph $G$ of order $n$ with $m$ edges, if there exists an edge $e \in$ $E(G)$ such that $G-e$ is a tree with $d(G-e) \geq 5$, then $S_{2}(G)<m(G)+3$.

Let $u v w u$ be a triangle and $T_{a, b, c}$ be the graph obtained by attaching $a, b, c$ pendent vertices to $u, v, w$, respectively, where $a+b+c=n-3$ and $a \geq b \geq c \geq 0$. Note that $T_{n-3,0,0} \cong$ $G_{n, n}$. Denote by $Q_{a, b}$ the graph obtained by attaching $a$ and $b$ pendent vertices to two nonadjacent vertices of a quadrangle, respectively, where $a+b=n-4$ and $a \geq b \geq 0 . T_{a, b, c}$ and $Q_{a, b}$ are illustrated in Figure 4.

Lemma 3.2 For $T_{a, b, c}, S_{2}\left(T_{a, b, c}\right) \leq m\left(T_{a, b, c}\right)+3$ with equality if and only if $a=n-3$ (that is, $\left.T_{a, b, c} \cong G_{n, n}\right)$. 

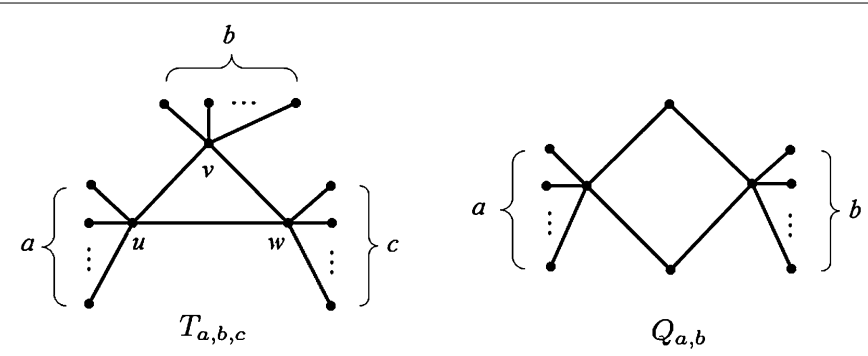

Figure 4 Two unicyclic graphs of order $\boldsymbol{n}$. $T_{a, b, c}$ is a unicyclic graph of order $n$ obtained by attaching $a, b, c$ pendent vertices to three vertices of a triangle $u v w$, respectively, and $Q_{a, b}$ is a unicyclic graph of order $n$ obtained by attaching $a$ and $b$ pendent vertices to two non-adjacent vertices of a quadrangle, respectively.

Proof Note that the matrix $1 \cdot I_{n}-L\left(T_{a, b, c}\right)$ has $a, b$ and $c$ different identical rows. So the multiplicity of eigenvalue 1 is at least $n-6$. Let $\lambda_{1} \geq \lambda_{2} \geq \lambda_{3} \geq \lambda_{4} \geq \lambda_{5}>\lambda_{6}=0$ be the other six eigenvalues of $L\left(T_{a, b, c}\right)$. Then we have $\lambda_{1}+\lambda_{2}+\lambda_{3}+\lambda_{4}+\lambda_{5}=n+6$ since $\sum_{i=1}^{i=n} \mu_{i}=2 m(G)$. For $c \geq 2, T_{a, b, c}$ contains $T_{2,2,2}$ as a subgraph. Then by Lemma 2.7 we have $\lambda_{3} \geq \mu_{3}\left(T_{2,2,2}\right)=3.0$. Therefore, $S_{2}\left(T_{a, b, c}\right)=\lambda_{1}+\lambda_{2}=n+6-\left(\lambda_{3}+\lambda_{4}+\lambda_{5}\right)<n+6-\lambda_{3} \leq$ $n+3=m\left(T_{a, b, c}\right)+3$, as required. For $c=1$ and $b \geq 4, T_{a, b, c}$ contains $T_{4,4,1}$ as a subgraph. Then by Lemma 2.7 we have $\lambda_{3} \geq \mu_{3}\left(T_{4,4,1}\right)=3.0$. That is, $S_{2}\left(T_{a, b, 1}\right)=n+6-\left(\lambda_{3}+\lambda_{4}+\lambda_{5}\right)<$ $n+6-\lambda_{3} \leq n+3=m\left(T_{a, b, c}\right)+3$, as required. If $c=1$ and $b=3$, then by Lemmas 2.1 and 2.8, we have $\mu_{1}\left(T_{a, 3,1}\right) \leq n-4+\frac{6}{n-5}$ and $\mu_{2}\left(T_{a, 3,1}\right) \leq \mu_{1}\left(L_{u}\left(T_{a, 3,1}\right)\right)=5.88$. It follows that $S_{2}\left(T_{a, 3,1}\right)=\mu_{1}\left(T_{a, 3,1}\right)+\mu_{2}\left(T_{a, 3,1}\right)<m\left(T_{a, 3,1}\right)+3$, as required. If $c=1$ and $b=2$, then by Lemmas 2.1 and 2.8, we have $\mu_{1}\left(T_{a, 2,1}\right) \leq n-3+\frac{5}{n-4}$ and $\mu_{2}\left(T_{a, 2,1}\right) \leq \mu_{1}\left(L_{u}\left(T_{a, 2,1}\right)\right)=5.05$. That is, $S_{2}\left(T_{a, 2,1}\right)=\mu_{1}\left(T_{a, 2,1}\right)+\mu_{2}\left(T_{a, 2,1}\right)<m\left(T_{a, 2,1}\right)+3$ for $n \geq 10$ (that is, $\left.a \geq 4\right)$, as required. A direct calculation shows that $S_{2}\left(T_{3,2,1}\right)<m\left(T_{3,2,1}\right)+3$ (or $S_{2}\left(T_{2,2,1}\right)<m\left(T_{2,2,1}\right)+3$ ). Similarly, if $c=1$ and $b=1$, then by Lemmas 2.1 and 2.8 , we have $\mu_{1}\left(T_{a, 1,1}\right) \leq n-2+\frac{4}{n-3}$ and $\mu_{2}\left(T_{a, 1,1}\right) \leq \mu_{1}\left(L_{u}\left(T_{a, 1,1}\right)\right)=4.30$. That is, $S_{2}\left(T_{a, 1,1}\right)=\mu_{1}\left(T_{a, 1,1}\right)+\mu_{2}\left(T_{a, 1,1}\right)<m\left(T_{a, 1,1}\right)+3$, for $n \geq 9$ (that is, $a \geq 4$ ), as required. A direct calculation shows that $S_{2}\left(T_{3,1,1}\right)<m\left(T_{3,1,1}\right)+3$ (or $S_{2}\left(T_{2,1,1}\right)<m\left(T_{2,1,1}\right)+3$ or $\left.S_{2}\left(T_{1,1,1}\right)<m\left(T_{1,1,1}\right)+3\right)$. Next, we assume $c=0$. By an elementary calculation, we have $\phi\left(T_{a, b, 0}, \lambda\right)=\lambda(\lambda-1)^{n-5} f_{2}(\lambda)$, where $f_{2}(\lambda)=\lambda^{4}-(n+5) \lambda^{3}+$ $(5 n+a b+7) \lambda^{2}-(7 n+2 a b+3) \lambda+3 n$. Let $x_{1} \geq x_{2} \geq x_{3} \geq x_{4}$ be the roots of $f_{2}(\lambda)=0$. Then

$$
\begin{aligned}
& x_{1}+x_{2}+x_{3}+x_{4}=n+5, \\
& x_{1} x_{2}+x_{1} x_{3}+x_{1} x_{4}+x_{2} x_{3}+x_{2} x_{4}+x_{3} x_{4}=5 n+a b+7, \\
& x_{2} x_{3} x_{4}+x_{1} x_{3} x_{4}+x_{1} x_{2} x_{4}+x_{1} x_{2} x_{3}=7 n+2 a b+3 .
\end{aligned}
$$

If

$$
x_{1}+x_{2}=n+3
$$

then, by (3.1), we have

$$
x_{3}+x_{4}=2 \text {. }
$$

From (3.2)-(3.5) it follows that

$$
x_{1} x_{2}+x_{3} x_{4}=3 n+a b+1,
$$




$$
(n+3) x_{3} x_{4}+2 x_{1} x_{2}=7 n+2 a b+3 .
$$

By (3.6) and (3.7), we have

$$
x_{3} x_{4}=1
$$

Combining (3.5) and (3.8), we have

$$
x_{3}=x_{4}=1 \text {. }
$$

Then $f_{2}(1)=-2 a b=0$, which implies that $b=0$. Therefore, if $b \geq 1$, then $S_{2}\left(T_{a, b, 0}\right)<$ $m\left(T_{a, b, 0}\right)+3$. A direct calculation shows that $S_{2}\left(T_{n-3,0,0}\right)=m\left(T_{n-3,0,0}\right)+3$. This completes the proof.

Lemma 3.3 For $Q_{a, b}, S_{2}\left(Q_{a, b}\right)<m\left(Q_{a, b}\right)+3$.

Proof By a direct calculation, we have $\phi\left(Q_{a, b}, \lambda\right)=\lambda(\lambda-1)^{n-6} f_{3}(\lambda)$, where $f_{3}(\lambda)=(\lambda-$ $2)\left(\lambda^{4}-(n+4) \lambda^{3}+(5 n+a b+1) \lambda^{2}-(6 n+2 a b-2) \lambda+2 n\right)$. Let $\lambda_{1} \geq \lambda_{2} \geq \lambda_{3} \geq \lambda_{4} \geq \lambda_{5}>0$ be the five roots of $f_{3}(\lambda)=0$. Then we have $\lambda_{1}+\lambda_{2}+\lambda_{3}+\lambda_{4}+\lambda_{5}=n+6$ since $\sum_{i=1}^{i=n} \mu_{i}=2 m(G)$. For $b \geq 1, Q_{a, b}$ contains $Q_{1,1}$ as a subgraph. Then by Lemma 2.7 we have $\lambda_{3} \geq \mu_{3}\left(Q_{1,1}\right)=2$ and $\lambda_{4} \geq \mu_{4}\left(Q_{1,1}\right)=1.26$. Therefore, $S_{2}\left(Q_{a, b}\right)=\lambda_{1}+\lambda_{2}=n+6-\lambda_{3}-\lambda_{4}-\lambda_{5}<n+3$. In what follows, we assume $b=0$. Since $f_{3}(1)=0$, we can rewrite $\phi\left(Q_{a, 0}, \lambda\right)=\lambda(\lambda-1)^{n-5} f_{4}(\lambda)$, where $f_{3}(\lambda)=(\lambda-1) f_{4}(\lambda)$. Let $\lambda_{1}^{\prime} \geq \lambda_{2}^{\prime} \geq \lambda_{3}^{\prime} \geq \lambda_{4}^{\prime}>0$ be the four roots of $f_{4}(\lambda)=0$. Then we have $\lambda_{1}^{\prime}+\lambda_{2}^{\prime}+\lambda_{3}^{\prime}+\lambda_{4}^{\prime}=n+5$ since $\sum_{i=1}^{i=n} \mu_{i}=2 m(G)$. For $a \geq 1, Q_{a, 0}$ contains $Q_{1,0}$ as a subgraph. Then by Lemma 2.7 we have $\lambda_{3}^{\prime} \geq \mu_{3}\left(Q_{1,0}\right)=2$. Thus, $S_{2}\left(Q_{a, b}\right)=\lambda_{1}^{\prime}+\lambda_{2}^{\prime}=n+5-\lambda_{3}^{\prime}-\lambda_{4}^{\prime}<$ $n+3$. If $a=0$, then $S_{2}\left(Q_{a, b}\right)=S_{2}\left(C_{4}\right)<m\left(C_{4}\right)+3$ by a straight calculation. This completes the proof.

Now, we come to the main results of this paper.

Theorem 3.4 For any unicyclic graph $G, S_{2}(G) \leq m(G)+3$ with equality if and only if $G \cong T(n-3,0,0)$.

Proof For any unicyclic graph $G$, we assume that $C_{k}=v_{1} v_{2} \cdots v_{k} v_{1}$ is the unique cycle in $G$ (for some $k$ ) and $G$ has the form $U\left(R_{1}, \ldots, R_{k}\right)$. For $k \geq 5, G-v_{1} v_{2}$ is a tree with $d\left(G-v_{1} v_{2}\right) \geq 5$. Then by Lemma 3.1 we have $S_{2}(G)<m(G)+3$. We now consider the following two cases.

Case 1. $k=4$.

Let $C_{4}=v_{1} v_{2} v_{3} v_{4} v_{1}$ be the unique cycle in $G$. If there exist $e\left(v_{i}\right) \geq 2$, say $e\left(v_{1}\right) \geq 2$, then $G-v_{1} v_{2}$ is a tree with $d\left(G-v_{1} v_{2}\right) \geq 5$; if there are two adjacent vertices in $C_{4}=v_{1} v_{2} v_{3} v_{4} v_{1}$, say $v_{1}$ and $v_{2}$, such that $e\left(v_{1}\right) \geq 1$ and $e\left(v_{2}\right) \geq 1$, then $G-v_{1} v_{2}$ is a tree with $d\left(G-v_{1} v_{2}\right) \geq 5$. Then by Lemma 3.1 we have $S_{2}(G)<m(G)+3$. We now assume $G \cong Q_{a, b}$ (see Figure 4 ). Then the result follows from Lemma 3.3.

Case $2 . k=3$.

If $\max \left\{e\left(v_{1}\right), e\left(v_{2}\right), e\left(v_{3}\right)\right\} \geq 3$, say $e\left(v_{1}\right) \geq 3$, then $G-v_{1} v_{2}$ is a tree with $d\left(G-v_{1} v_{2}\right) \geq 5$; if there are two vertices in $C_{3}=v_{1} v_{2} v_{3} v_{1}$, say $v_{1}$ and $v_{2}$, such that $e\left(v_{1}\right)=2$ and $e\left(v_{2}\right) \geq 1$, then $G-v_{1} v_{2}$ is a tree with $d\left(G-v_{1} v_{2}\right) \geq 5$. Therefore, Lemma 3.1 implies that $S_{2}(G)<$ 
$m(G)+3$. We now assume $G \cong F_{1, t, n-2 t-3}(t \geq 1)$ or $G \cong T_{a, b, c}$. Then the result follows from Lemma 2.13 or Lemma 3.2 .

This completes the proof.

Competing interests

The authors declare that they have no competing interests.

\section{Authors' contributions}

$Y Z$ carried out the proofs of the main results in the manuscript. AC and $J L$ participated in the design of the study and drafted the manuscript. All the authors read and approved the final manuscript.

\section{Author details}

'School of Applied Mathematics, Xiamen University of Technology, Xiamen, Fujian, P.R. China. ${ }^{2}$ Center for Discrete Mathematics, Fuzhou University, Fuzhou, Fujian, P.R. China. ${ }^{3}$ School of Mathematics and Statistics, Minnan Normal University, Zhangzhou, Fujian, P.R. China.

\section{Acknowledgements}

The authors would like to thank the anonymous referees for their constructive corrections and valuable comments on this paper, which have considerably improved the presentation of this paper. This project is supported by NSF of China (Nos. 11471077, 11301440), China Postdoctoral Science Foundation (No. 2014M551831), NSF of Fujian (No. 2014J01020), the Foundation to the Educational Committee of Fujian (JA13240, JA15381, JB13155).

Received: 31 March 2015 Accepted: 23 August 2015 Published online: 17 September 2015

\section{References}

1. Brouwer, A, Haemers, W: Spectra of Graphs. Springer, New York (2012)

2. Godsil, C, Royle, G: Algebraic Graph Theory. Springer, New York (2001)

3. Fiedler, M: Algebraic connectivity of graphs. Czechoslov. Math. J. 23, 298-305 (1973)

4. Anderson, W, Morley, T: Eigenvalues of the Laplacian of a graph. Linear Multilinear Algebra 18, $141-145$ (1985)

5. Guo, J-M: On the second largest Laplacian eigenvalue of trees. Linear Algebra Appl. 404, 251-261 (2005)

6. Li, J, Guo, J-M, Shiu, WC: On the second largest Laplacian eigenvalue of graphs. Linear Algebra Appl. 438, 2438-2446 (2013)

7. Liu, M, Liu, B, Cheng, B: Ordering (signless) Laplacian spectral radii with maximum degree of graphs. Discrete Math. 338, 159-163 (2015)

8. Merris, R: A note on the Laplacian graph eigenvalues. Linear Algebra Appl. 285, 33-35 (1990)

9. Zhang, X-D, Li, J: The two largest Laplacian eigenvalues of trees. J. Univ. Sci. Technol. China 28, 513-518 (1998)

10. Du, Z, Zhou, B: Upper bounds for the sum of Laplacian eigenvalues of graphs. Linear Algebra Appl. 436, 3672-3683 (2012)

11. Guan, M, Zhai, M, Wu, Y: On the sum of two largest Laplacian eigenvalue of trees. J. Inequal. Appl. 2014, 242 (2014)

12. Haemers, W, Mohammadian, A, Tayfeh-Rezaie, B: On the sum of Laplacian eigenvalues of graphs. Linear Algebra Appl. 432, 2214-2221 (2010)

13. Rocha, I, Trevisian, V: Bounding the sum of the Laplacian graph eigenvalues of graphs. Discrete Appl. Math. 170 95-103 (2014)

14. Wang, S, Huang, Y, Liu, B: On the conjecture for the sum of the Laplacian graph eigenvalues. Math. Comput. Model. 56, 60-68 (2012)

15. Fritscher, E, Hoppen, C, Rocha, I, Trevisan, V: On the sum of the Laplacian eigenvalues of a tree. Linear Algebra Appl. 435, 371-399 (2011)

16. Fan, K: On a theorem of Wely concerning eigenvalues of linear transformations. Proc. Natl. Acad. Sci. USA 35, 652-655 (1949)

\section{Submit your manuscript to a SpringerOpen ${ }^{\circ}$ journal and benefit from:}

- Convenient online submission

Rigorous peer review

- Immediate publication on acceptance

- Open access: articles freely available online

- High visibility within the field

- Retaining the copyright to your article 